\title{
Brain Structural Features of Myotonic Dystrophy Type 1 and their Relationship with CTG Repeats
}

Ellen van der Plas ${ }^{\mathrm{a}, 1}$, Mark J. Hamilton ${ }^{\mathrm{b}, \mathrm{c}, 1, *}$, Jacob N. Miller ${ }^{\mathrm{a}}$, Timothy R. Koscik ${ }^{\mathrm{a}}$, Jeffrey D. Long ${ }^{\mathrm{a}, \mathrm{d}}$, Sarah Cumming ${ }^{\mathrm{c}}$, Julija Povilaikaite ${ }^{\mathrm{c}}$, Maria Elena Farrugia ${ }^{\mathrm{e}}$, John McLean ${ }^{\mathrm{f}}$, Ravi Jampana ${ }^{\mathrm{f}}$, Vincent A. Magnotta ${ }^{\mathrm{g}}$, Laurie Gutmann ${ }^{\mathrm{h}}$, Darren G. Monckton ${ }^{\mathrm{c}}$ and Peggy C. Nopoulos ${ }^{\mathrm{a}}$ ${ }^{a}$ Department of Psychiatry, University of Iowa Hospital and Clinics, Iowa City, IA, USA

${ }^{\mathrm{b}}$ West of Scotland Clinical Genetics Service, Queen Elizabeth University Hospital, Glasgow, UK

${ }^{\mathrm{c}}$ Institute of Molecular, Cell and Systems Biology, College of Medical, Veterinary and Life Sciences, University of Glasgow, Glasgow, UK

${ }^{\mathrm{d}}$ Department of Biostatistics, University of Iowa, College of Public Health, Iowa City, IA, USA

${ }^{\mathrm{e}}$ Department of Neurology, Institute of Neurological Sciences, Queen Elizabeth

University Hospital, Glasgow, UK

${ }_{\mathrm{f}}^{\mathrm{f}}$ Department of Neuroradiology, Institute of Neurological Sciences, Queen Elizabeth

University Hospital, Glasgow, UK

$\mathrm{g}^{\mathrm{g}}$ Department of Radiology, University of Iowa Hospital and Clinics, Iowa City, IA, USA

${ }^{\mathrm{h}}$ Department of Neurology, University of Iowa Hospital and Clinics, Iowa City, IA, USA

\begin{abstract}
.
Background: Few adequately-powered studies have systematically evaluated brain morphology in adult-onset myotonic dystrophy type 1 (DM1).

Objective: The goal of the present study was to determine structural brain differences between individuals with and without adult-onset DM1 in a multi-site, case-controlled cohort. We also explored correlations between brain structure and CTG repeat length.

Methods: Neuroimaging data was acquired in 58 unaffected individuals (29 women) and 79 individuals with DM1 (50 women). CTG repeat length, expressed as estimated progenitor allele length (ePAL), was determined by small pool PCR. Statistical models were adjusted for age, sex, site, and intracranial volume (ICV).

Results: ICV was reduced in DM1 subjects compared with controls. Accounting for the difference in ICV, the DM1 group exhibited smaller volume in frontal grey and white matter, parietal grey matter as well as smaller volume of the corpus callosum, thalamus, putamen, and accumbens. In contrast, volumes of the hippocampus and amygdala were significantly larger in DM1. Greater ePAL was associated with lower volumes of the putamen, occipital grey matter, and thalamus. A positive ePAL association was observed for amygdala volume and cerebellar white matter.

Conclusions: Smaller ICV may be a marker of aberrant neurodevelopment in adult-onset DM1. Volumetric analysis revealed morphological differences, some associated with CTG repeat length, in structures with plausible links to key DM1 symptoms including cognitive deficits and excessive daytime somnolence. These data offer further insights into the basis of CNS disease in DM1, and highlight avenues for further work to identify therapeutic targets and imaging biomarkers.
\end{abstract}

Keywords: Myotonic dystrophy, magnetic resonance imaging, neuroanatomy

\footnotetext{
${ }^{1}$ These authors contributed equally to the manuscript

*Correspondence to: Mark J. Hamilton, PhD, Current address: Department of Clinical Genetics, City Hospital, Hucknall
}

Road, Nottingham NG5 1PB, UK. E-mail: markhamilton1@nhs. net. 


\section{INTRODUCTION}

Myotonic dystrophy type 1 (DM1) is a dominantly inherited, multisystem disorder, resulting from expansion of a CTG trinucleotide repeat in the 3'-untranslated region of $D M P K$ [1]. The clinical phenotype is widely variable, with common symptoms including skeletal muscle weakness and myotonia, cataracts, cardiac conduction defects, and male hypogonadism. Involvement of the CNS underlies additional symptoms such as cognitive deficits, excessive daytime somnolence, apathy, and impaired social functioning [2]. CNS symptoms are reported frequently by patients [3], and may greatly impact quality of life $[4,5]$, relationships with caregivers [6] and engagement with medical care [7]. With the advent of clinical trials of potential diseasemodifying therapies for DM1, expert working groups have highlighted the need to improve understanding of the mechanisms underlying CNS disease, both to identify neuroimaging biomarkers and reveal potential targets for therapy $[8,9]$.

MRI studies in patients with DM1 have identified morphometric abnormalities in the brain, including global volume loss, ventricular dilation, white matter lesions, and disruptions of white matter microstructure evident in diffusion tensor imaging [10]. According to several studies that used voxelbased morphometry (VBM), global volume loss may be driven by grey matter atrophy in the cerebral cortex and subcortical structures [11-13]. Some VBM studies have also demonstrated associations between focal brain morphology and cognitive abilities [14, 15], and/or CTG repeat length [16, 17], although others found no association $[12,13]$.

Several limitations of preceding neuroimaging studies should be considered. First, previous studies have generally included fewer than 40 affected individuals, limiting the power to detect meaningful abnormalities in brain morphology $[11,13,15$, 18, 19]. Second, few studies have validated the VBM findings with alternative approaches that enable comprehensive exploration of global and regional grey and white matter structural differences between patients and controls [18]. Third, previous studies have relied on traditional methods to measure CTG repeat expansion which fail to take account of agedependent, expansion-biased mosaicism in somatic cells [20]. Finally, some existing studies have combined congenital DM1 (CDM), juvenile-onset DM1 (JDM), and adult-onset DM1 in a single sample [13, 17]. Individuals with CDM exhibit severe cogni- tive delay [21], while those with adult-onset DM1 typically have much milder impairment [22]. The diversity of CNS phenotypes in heterogeneous samples confounds analyses of structural correlations further.

To address these limitations, we harmonized neuroimaging protocols across two sites to obtain a large, homogeneous, case-controlled sample of individuals with adult-onset DM1. A volume-based brain parcellation approach was applied to characterize brain structures. Genotyping was conducted by smallpool PCR (SP-PCR) to estimate the progenitor allele length, which has been shown to improve clinical correlations compared to traditional methods [23].

\section{MATERIALS AND METHODS}

\section{Participants}

Individuals with DM1 were recruited to the University of Iowa from across the United States via advertisements through the Myotonic Dystrophy Foundation (MDF) and word of mouth. Unaffected participants were primarily recruited from the Iowa City area via advertisements. Some were unaffected spouses of affected participants. Recruitment took place between September 2014 and July 2017.

In Glasgow, UK, individuals with DM1 attending the annual review at the West of Scotland Clinical Genetics Service were invited to participate. Unaffected participants were recruited from affected subjects' families and from the Scottish Health Research Register (www.registerforshare.org) [24]. Participants in Glasgow were recruited between January 2016 and May 2017.

Exclusion criteria for all participants were: learning disability in childhood, a history of serious head injury, or a chronic neurological disorder other than DM1. Unaffected participants were additionally required to be without history of substance abuse, psychiatric disease, or major medical disease, including: heart disease, sleep disorder, vascular disease, uncontrolled hypertension, cancer, diabetes mellitus, lung disease, and autoimmune conditions.

Recruitment was targeted to adult-onset DM1 only. The Glasgow site recruited only patients who denied onset of DM1-specific symptoms before age 16, while the Iowa site included participants who denied symptoms before age 18 . The sample included seven individuals who had self-reported age of first onset before age 18 . 
Participants underwent genetic testing as part of the research study. All participants recruited into the DM1-affected group at the Glasgow site, and the majority at the Iowa site (93\%), had also undergone genetic testing prior to participation and were aware of their genetic status. A subset of participants in Iowa were at-risk for DM1, but had not undergone predictive testing $(n=11)$. At-risk individuals in the Iowa sample who were determined to have CTG repeat length $>50$ were included in the DM1 group $(n=4)$. The remainder had CTG repeat lengths in the nondisease associated range, and were included in the control group $(n=7)$. Research staff, clinicians and scientists involved in this study remained blind to the genetic status of at-risk individuals. All data were de-identified and all participants at the Iowa site consented to non-disclosure of genetic results obtained as part of the study. Control participants recruited in Glasgow were not genotyped for CTG repeat length, but none was at risk of DM1 by family history, and none exhibited overt signs of any neurological disorder. Control participants in Iowa were genotyped for CTG repeat length, to confirm unaffected status.

All participants gave written, informed consent prior to enrolling in the protocol in accordance to the Declaration of Helsinki. The study was approved by the University of Iowa's Institutional Review Board or the West of Scotland Research Ethics Committee (Reference: WOS 15/WS/0189).

\section{Muscle impairment rating scale (MIRS)}

Severity of muscle weakness, expressed as muscle impairment rating scale (MIRS) [25], was determined by examination by a neurologist or other specialist experienced in DM1.

\section{Image acquisition}

Iowa participants who participated before June $2016(n=52)$ were scanned using a 3T Siemens Trio TIM (Siemens AG, Munich, Germany; 12channel head coil), and those who participated after June $2016(n=27)$ were scanned on 3T General Electric Discovery MR750w (GE Medical Systems, Chicago, IL, 16-channel head and neck coil). Anatomical T1-weighted images were acquired as follows for Siemens (GE parameters in parentheses): coronal MPRAGE (BRAVO), TR = $2300(8.392) \mathrm{ms}$, $\mathrm{TE}=2.82(3.184) \mathrm{ms}, \mathrm{TI}=900(450) \mathrm{ms}$, flip angle $=$ $10(12)^{\circ}, \mathrm{FOV}=282 \times 282 \times 264 \mathrm{~mm}$, matrix $=256$ $\times 256 \times 240$. Parameters for T2-weighted images were: coronal, TR $=4800(3000) \mathrm{ms}, \mathrm{TE}=430(85$. 925) $\mathrm{ms}, \mathrm{FOV}=256 \times 256 \times 224 \mathrm{~mm}$, matrix $=256$ $\times 256 \times 160$.

In Scotland, imaging was carried out on a $3 \mathrm{~T}$ Siemens Prisma MRI scanner (Software version: VE11B. Erlangen, Germany, 20-channel head and neck coil). T1-weighted parameters: coronal MPR AGE, $\mathrm{TR}=2300 \mathrm{~ms}, \mathrm{TE}=2.01 \mathrm{~ms}, \mathrm{TI}=900 \mathrm{~ms}$, flip angle $=10^{\circ}, \mathrm{FOV}=282 \times 282 \times 211 \mathrm{~mm}$, matrix $=$ $256 \times 256 \times 192$. T2-weighted parameters coronal SPACE, TR $=3200 \mathrm{~ms}, \mathrm{TE}=407 \mathrm{~ms}, \mathrm{FOV}=282 \times$ $198 \times 194 \mathrm{~mm}$, matrix $=256 \times 180 \times 176$.

\section{Image processing}

Bias field inhomogeneity was corrected using the N4 algorithm implemented in Advanced Normalization Tools software [26]. Images were processed using the BRAINSAutoWorkup pipeline which optimizes tissue classification through an iterative framework and produces robust parcellation of brain regions in a multi-site setting [27]. BRAINSAutoWorkup labels brain regions using a multi-atlas, similarity-weighted, majority-vote procedure (joint label fusion, [28]) using a set of expert-segmented templates adapted from the Desikan-Killiany atlas [29]. Brain regions include cortical and subcortical regions, separated by hemispheres and tissue type (gray or white matter) where appropriate. Residual inter-scanner variation was harmonized using an empirical Bayesian approach [30, 31] as implemented by the ez.combat toolbox in $\mathrm{R}$ [32]. We confirmed that scanner did not predict regional volume by conducting Kolmogorov-Smirnov tests (Supplementary Table 1) and visualizing the empirical cumulative distributions for each scanner across ROIs and groups (Supplementary Figure 1). Statistical analyses were performed on the harmonized neuroimaging data.

\section{Measurement of CTG repeat length}

Genotyping of CTG repeat in DM1 participants was completed by SP-PCR [33]. For each patient, four reactions were completed, each using $300 \mathrm{pg}$ blood genomic DNA template. CTG repeat lengths were estimated by comparison against DNA fragments of known length in the molecular weight marker, using CLIQS software (TotalLabs UK Ltd.). The lower boundary of the expanded molecules in SP-PCR was used to estimate the 
inherited or progenitor allele length (ePAL) [34], which is the major determinant of age at symptom onset [23].

\section{Statistical analyses}

It is important to account for intracranial volume (ICV) when comparing regions between groups, because regions scale with ICV. The goal of correction is to transform the region of interest (ROI) such that it is no longer related to ICV, which requires accounting for non-linear relationships. The power-proportion method (PPM) divides volume by $\beta$, where $\beta$ is estimated from a non-linear regression model, $R O I=\alpha I C V^{\beta}$ [35]. We estimated $\beta$ for each ROI, and divided ROI by $\mathrm{ICV}^{\beta}$. Each ratio was subsequently standardized by subtracting out the grand mean, and dividing by the SD. The efficacy of detrending was checked by running linear regression models predicting the adjusted ROI from ICV. None of the estimates were statistically significant (unadjusted, lowest $p$-value $=0.647$; see Supplementary Figure 2).

The multivariable linear regression models to examine group differences included the harmonized, standardized, PPM-adjusted ROI volumes as the dependent variable, and group, age, sex, and site as predictors:

$$
Z_{i}=\gamma_{0}+\gamma_{1} \operatorname{group}_{i}+\gamma_{2} \operatorname{age}_{i}+\gamma_{3} \operatorname{sex}_{i}+\gamma_{4} \text { site }_{i}+e_{i},
$$

In this equation, $Z_{i}$ is the adjusted ROI volume for the $i$ th participant $(i=1, \ldots, N)$. Group, sex, and site were expressed as numeric factors. It was assumed that the $e_{i}$ were normally distributed with zero mean and non-zero variance.

The models for ePAL considered only the DM1 group:

$$
Z_{i}=\gamma_{0}+\gamma_{1} e P A L_{i}+\gamma_{2} \text { age }_{i}+\gamma_{3} \text { sex }_{i}+\gamma_{4} \text { site }_{i}+e_{i},
$$

Group and ePAL estimates are presented as adjusted means normalized to the standard deviation. Inference was based on the $99 \%$ confidence interval (CI).

\section{RESULTS}

\section{Sample}

The pooled sample included 58 unaffected individuals and 79 individuals with DM1. The Iowa site contributed 38 unaffected individuals and 41 individuals with DM1, and the Glasgow site contributed 20 unaffected individuals and 38 individuals with DM1 (Table 1). The unaffected group included equal numbers of men $(n=29)$ and women $(n=29)$, while the DM1 group included significantly more women $(n=50)$ than men $(n=29), \chi^{2}(1)=6.0, p=0.02$. Note that statistical models are adjusted for sex. Average age at evaluation was 46 years old $(\mathrm{SD}=13$ years), with no significant difference between groups, $t_{(134)}=0.5, p=0.6$. ePAL values in the DM1 group ranged from 55 to 572 CTG repeats (Fig. 1), and self-reported age at onset of DM1 symptoms was on average 33 years old $(\mathrm{SD}=12$ years) (Table 1$)$.

\section{Brain morphology}

Sex was a significant predictor of intracranial volume (ICV), with women exhibiting lower ICV than men, $t_{(132)}=9.6, p<0.0001$. Age did not predict ICV $(p=0.1)$. The group coefficient for ICV was significant $\left(t_{(132)}=-4.1, p<0.0001\right)$, with mean ICV being $7.6 \%$ lower in the DM1 group compared with unaffected individuals (Fig. 2A).

Both sex and age were significant predictors of cerebrospinal fluid volume, $t_{(132)}=2.8, p<0.01$, and $t_{(132)}=9.6, p<0.00001$, respectively. The group coefficient was also statistically significant $t_{(132)}=3.8$, $p<0.001)$. Individuals affected with DM1 had approximately $11 \%$ greater CSF volume than unaffected individuals (Fig. 2A).

Compared with unaffected individuals, the DM1affected group exhibited significantly lower cerebral volume $\left(t_{(132)}=-4.2, p<.0001\right)$, evidently driven mostly by cerebral grey matter $\left(t_{(132)}=-4.4\right.$, $p<0.0001)$. Cerebellar white matter was significantly reduced in DM1 as well, $t_{(132)}=-2.7, p=0.01$. Across cerebral lobes, the DM1 group exhibited lower volume in frontal grey $\left(t_{(132)}=-4.4, p<0.0001\right)$ and white matter $\left(t_{(132)}=-2.4, p=0.02\right)$, parietal grey matter $\left(t_{(132)}=-4.2, p<0.0001\right)$, as well as corpus callosum $\left(t_{(132)}=-3.5, p<0.0001\right)$. Subcortically, affected individuals exhibited lower volumes of puta$\operatorname{men}\left(t_{(132)}=-2.1, p=0.04\right)$, accumbens $\left(t_{(132)}=-2.3\right.$, $p=0.03)$, and thalamus $\left(t_{(132)}=-2.8, p=0.01\right)$. By contrast, hippocampus $\left(t_{(132)}=2.4, p=0.02\right)$ and amygdala volume $\left(t_{(132)}=5.4, p<0.00001\right)$ were larger in individuals affected with DM1 (Fig. 2A and $2 \mathrm{~B}$ ). Unadjusted parcellation volumes in these regions also appeared larger in the DM1-affected group prior to standardization (Supplementary Figure 3). 


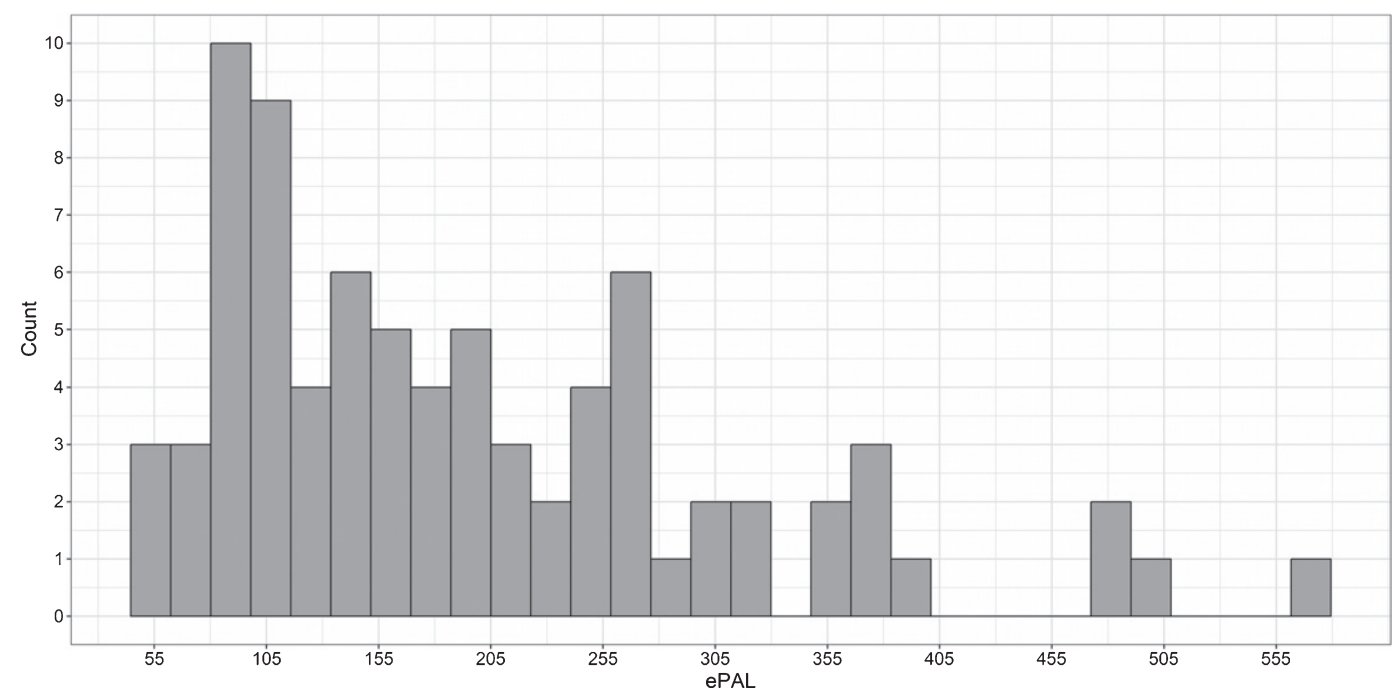

Fig. 1. Distribution of CTG repeat length (expressed as ePAL) among individuals with DM1 in the sample.

Table 1

Site characteristics

\begin{tabular}{|c|c|c|c|c|c|c|}
\hline & & & & ols & & \\
\hline & & & Scotland & Iowa & Scotland & Iowa \\
\hline Sample & $\mathrm{N}$ & & 20 & 38 & 38 & 41 \\
\hline Sex & $\mathrm{N}$ & Males & 12 & 17 & 17 & 12 \\
\hline & & Females & 8 & 21 & 21 & 29 \\
\hline Age at evaluation & & Mean (SD) & $46.1(13.1)$ & $44.6(13.7)$ & $47.1(13.2)$ & $45.4(11.7)$ \\
\hline Age at symptom onset & & Mean (SD) & - & - & $30.5(13.4)$ & $35.4(10.3)$ \\
\hline Muscle impairment & $\mathrm{N}$ & MIRS 1 & - & - & 3 & 12 \\
\hline & & MIRS 2 & - & - & 6 & 19 \\
\hline & & MIRS 3 & - & - & 10 & 5 \\
\hline & & MIRS 4 & - & - & 18 & 5 \\
\hline & & MIRS 5 & - & - & 1 & 0 \\
\hline ePAL & & Range & - & - & $56-572$ & $55-501$ \\
\hline & & Median & - & - & 211 & 131 \\
\hline & & Interquartile range & - & - & $152-269$ & $88-215$ \\
\hline
\end{tabular}

Analyses indicated a negative coefficient for ePAL when predicting adjusted putamen volume $\left(t_{(132)}=-2.9, p=0.01\right)$, occipital grey matter $\left(t_{(132)}=-2.5, p=0.01\right)$, and thalamus $\left(t_{(132)}=-2.0\right.$, $p=0.05$; Fig. 3). A positive ePAL coefficient was observed for amygdala volume $\left(t_{(132)}=2.2, p=0.03\right)$, and cerebellar white matter $\left(t_{(132)}=1.98, p=0.05\right)$ (Fig. 3).

\section{DISCUSSION}

The present study constitutes the largest neuroimaging study in DM1 to date, demonstrating that multi-site neuroimaging is a feasible, powerful approach to elucidate mechanisms of CNS disease in DM1. We restricted our sample to adult- onset DM1 to limit confounders associated with the markedly diverse clinical continuum, such as intellectual deficits that are evident in congenital and juvenile onset DM1 [21]. We also added a comprehensive, volumetric parcellation approach to the existing literature on brain morphology in DM1, and identified regional structural brain abnormalities in individuals with adult-onset DM1. We demonstrated that variation in volume of the putamen, occipital gray matter, thalamus, and amygdala was significantly predicted by CTG repeat expansion, strengthening the link between regional abnormalities and diseasespecificity.

In line with earlier work [15, 36-39], we replicate evidence of lower ICV in individuals with DM1. ICV is considered a marker of maximal brain growth and is largely determined by the age of 10 years, irrespec- 


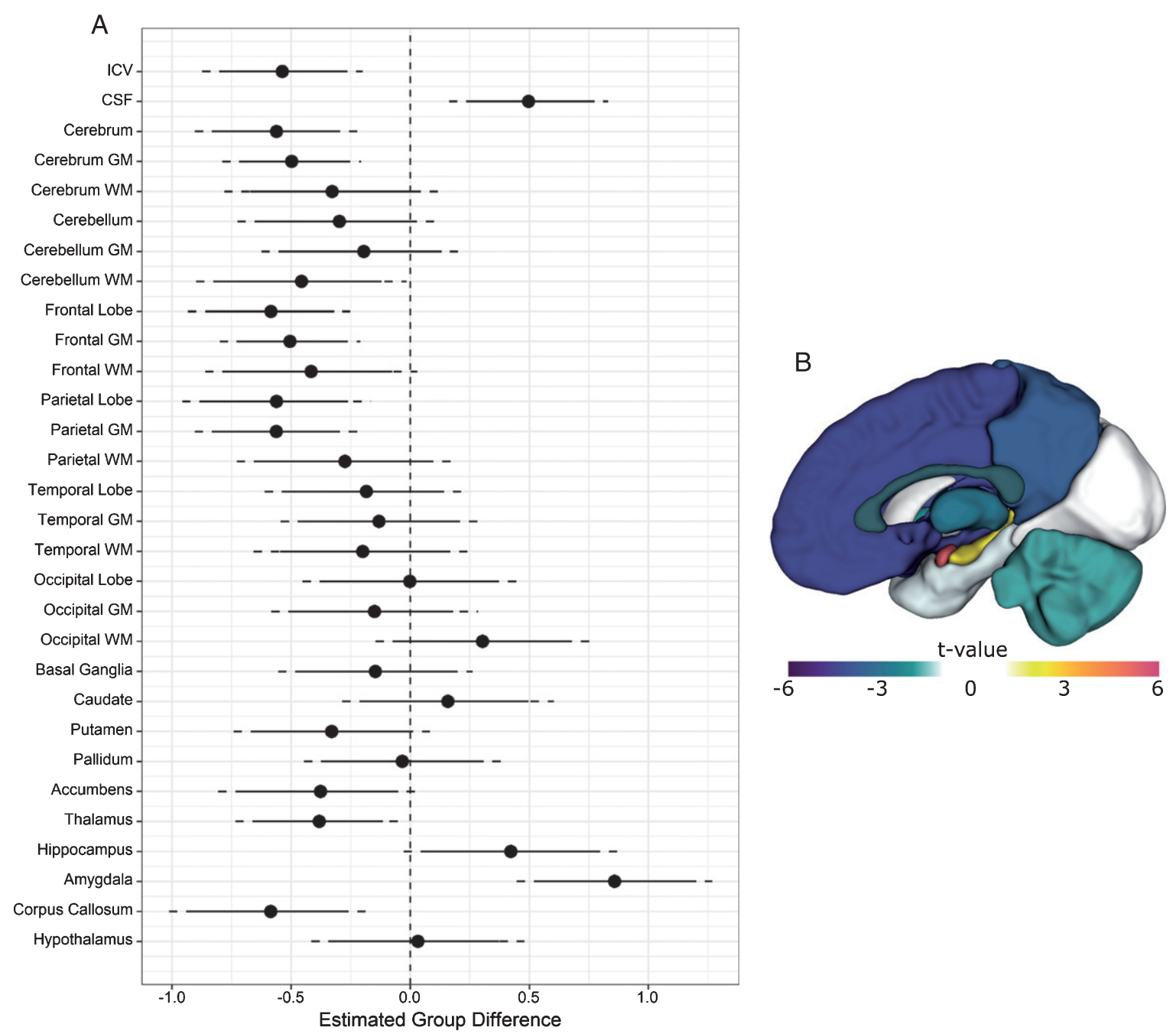

Fig. 2. Structural brain differences between DM1 patients and unaffected adults. Panel A: The circles represent estimated group differences adjusted for sex, age and site in SD units (x-axis) for regional volume (y-axis). The vertical, dashed line marks 0, i.e., no group difference. The solid lines represent the $95 \%$ confidence limits, and the dashed lines the $99 \%$ confidence limits. Negative differences indicate that the DM1 group had smaller volumes than did controls, while positive differences indicate that the DM1 group had larger volumes than controls. Panel B: Summary of group differences in regional volumes between individuals with and without DM1 after ICV adjustment. The colors correspond with the magnitude of the $t$-values of group differences (see color scale). Cool colors indicate that the DM1 group had lower volume than the unaffected group, while hot colors indicate the opposite pattern.

tive of subsequent changes in the brain parenchyma [40]. The finding of reduced ICV is therefore particularly notable in our cohort, in which recruitment was targeted to the adult-onset form of DM1. While it must be acknowledged that timing of onset of symptoms is a somewhat subjective measure, and it cannot be excluded that some subjects could have manifested subtle features of DM1 in childhood, this finding does imply that presence of the expanded CTG repeat has an effect on CNS structure prior to the onset of overt classical DM1 symptoms.
The cause of restricted brain volume in DM1 is not known. It could be hypothesised to result from a variety of mechanisms, including abnormal development, differences in cell volume, reduced proliferation, or early loss of neurons [41, 42]. In patients with schizophrenia, who show more subtle reduction in ICV [43], reduced grey matter volume and synaptic density is believed to occur due to an excess of synaptic pruning [44], the physiological process of neural remodelling that typically occurs from childhood through adolescence. Early-onset, excessive pruning 


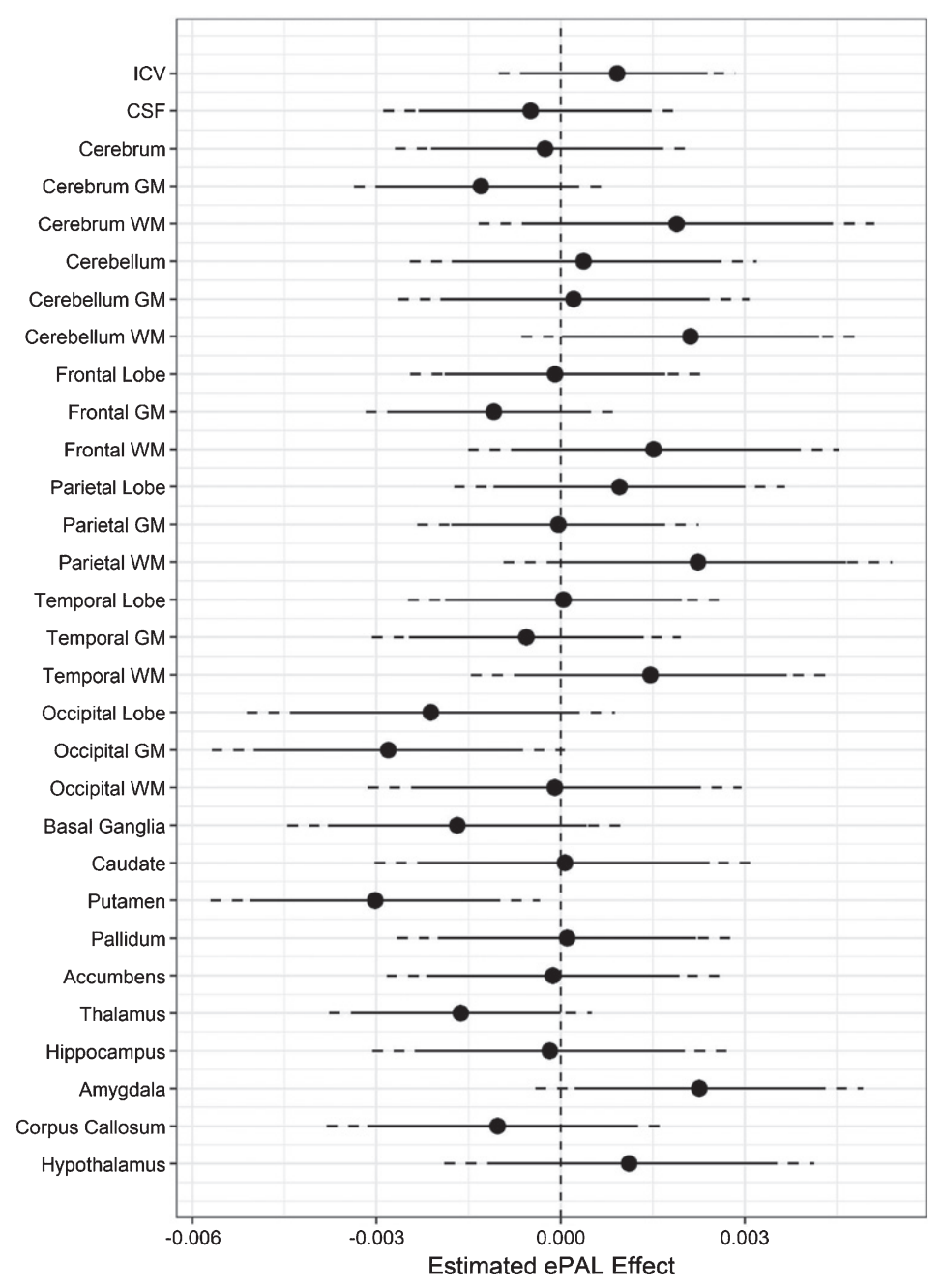

Fig. 3. Association between regional brain volume and ePAL in DM1. The circles represent estimated coefficients (circles) for the relationship between ePAL and regional volume (y-axis), adjusted for sex, age, and site in SD units (x-axis) among individuals with DM1. The vertical, dashed lines marks 0, i.e., no effect of ePAL. The solid lines represent $95 \%$ confidence limits, and the dashed lines represent $99 \%$ confidence limits.

could conceivably represent an additional mechanism affecting maximum ICV growth in DM1. Of note, it is unclear whether the modest reduction of IQ observed in cohorts with adult-onset DM1 entirely represents a neurodegenerative process [45], or may have its roots in abnormal brain development. Neurodevelopmental aspects of adult-onset DM1 therefore represent a key area for further work to improve understanding of CNS disease.

Regional brain volume was found to be reduced in DM1-affected subjects, while CSF volume was greater in the DM1 group than the unaffected group. Studies on the aging brain or in neurodegenerative disorders have demonstrated that atrophy coincides with increased ventricular spaces and greater CSF volume [46, 47]. Regional volume reductions in DM1 are thought to be the result of atrophy $[10,19]$, therefore the observed increase in CSF is to be expected. We observed reduced volume of frontal and parietal lobes, as well as substantial loss in subcortical structures including thalamus and putamen, consistent with the findings of previous studies in smaller cohorts using alternative neuroimaging techniques [11-13, 18]. The functional impact of structural changes observed is not clear from the available data, so the need to explore clinical correlations of brain morphological abnormalities remains. The functional impact of subcortical volume loss is of particular interest, given the roles of these structures in maintaining wakefulness, regulating sleep architecture, 
and in cognitive processing [48], which could clearly be relevant to elements of the DM1 phenotype.

Variation in putamen, thalamus and occipital grey matter volume was negatively related to the size of the CTG repeat expansion (adjusting for covariates), while amygdala volume was positively associated with CTG repeat length. These results support a direct association between these regional brain abnormalities and the primary genetic lesion in DM1. The positive association between CTG repeat length and cerebellar white matter volume is somewhat puzzling, given the observation that cerebellar white matter was significantly reduced in the DM1 group compared with the unaffected group.

The processes by which CTG expansion leads to structural brain change in DM1 are incompletely understood. Histopathology studies of brain suggest a complex phenotype, including widespread neuronal loss, presence of neurofibrillary tangles, hyalinisation of capillary walls and diffuse widening of perivascular spaces $[49,50]$. At the cellular level, CUG repeat mRNAs arising from the expanded repeat are seen to form discrete foci in the nuclei of DM1-affected neurons. Presence of these foci is associated with a dysregulation of alternative splicing affecting a wide range of mRNA targets [51], with the potential to impact a range of critical pathways and structural proteins in neural cells. For example, tau protein is subject to a shift in alternative splicing, favoring fetal isoforms [52]. These isoforms have lower affinity to bind and stabilize the microtubular cytoskeleton, and may disrupt axonal transport. Overlapping histological features between DM1 and Alzheimer dementia, including presence of neurofibrillary tangles, strongly supports a role of tau dysfunction in CNS pathogenesis [53]. Evidence for dysregulated splicing of the NMDA receptor subunit NMDARI [54] and altered expression of synaptic vesicle proteins [55] suggest a role for disruption of synaptic signalling also.

It is possible that different mechanisms are predominantly responsible for driving structural change in different regions of brain, which could account for variation in the degree of volumetric change and strength/direction of correlations with the CTG repeat between regions. Expansion-biased somatic instability of the CTG repeat in DM1 varies between tissues, and is generally seen to be greater in the tissues most affected by the disease, such as muscle [56]. Intriguingly, in the CNS, somatic instability is comparatively low in cerebellar cortex compared with frontal cerebral cortex [50], consistent with the relative severity of volume loss observed in these structures. Further studies of postmortem brain would be useful to gather further evidence for variation in pathology in different brain regions. Systematic measurement of somatic instability in specific structures would be particularly useful to evaluate this mechanism as a driver of CNS pathology, and hence possible target for therapy.

Larger volume of amygdala and hippocampus was an unexpected finding, particularly in the context of widespread volume loss. This observation is notable in the context of a previous study using VBM which reported reduced hippocampal volume in DM1 [39]. In addition to using a different parcellation approach, the present study included a sample around five times larger than the study by Weber and colleagues. It should also be noted that the observed increase in amygdala and hippocampal volumes was not an artefact of our standardization method, as the DM1 group exhibited larger unadjusted volumes in these regions than unaffected controls (see Supplementary Figure 3).

The cause of increased volume is unclear, but may relate to loss of mechanical growth restriction due to atrophy of adjacent structures, a compensatory change to preserve function in neuronal systems, abnormal growth driven by the molecular pathology of DM1, a combination of these factors, or other processes. The limited histological evidence available from previous studies suggest amygdala and hippocampus are not spared from the disease process, in that they exhibit typical hallmarks of DM1 including neurofibrillary tangles $[49,57]$. Interestingly, sleep disordered breathing has been linked to both regional increases and decreases in hippocampus volume in non-DM1 subjects, held to represent the acute and chronic effects of hypoxia respectively [58]. Further, emerging evidence suggests a functional link between amygdala and control of respiration, with stimulation of the amygdala seen to induce apnea [59]. Given the high prevalence of sleep disordered breathing in DM1, including presence of central apneas [60], clinical correlations with respiratory measures would be important to explore in future work. The amygdalo-striatal circuitry has further been implicated in motivational salience [61], raising questions about the potential role of amygdalo-striatal dysfunction in symptoms of depression, apathy, and anhedonia that are commonly observed among individuals with DM1 [62]. Current efforts to develop therapy for anxiety disorders, through manipulation of specific inhibitory neuron populations within 
the amygdala [63], could therefore yield useful therapeutic targets for a range of CNS symptoms in DM1.

A limitation of the present study is that volumetric analysis gives limited information regarding the structural or functional integrity of white matter. In contrast to some previous data [11], we found that volumetric changes were generally less marked in white matter structures compared with associated grey matter. Nonetheless, white matter pathology clearly represents an important component of the CNS structural landscape in DM1, as evidenced by the frequent occurrence of T2 hyperintense lesions [10], and widespread reduced fractional anisotropy on diffusion tensor imaging, even in normal-appearing white matter [64]. It remains uncertain to what extent white matter disease represents a primary manifestation of the DM1 disease process, versus Wallerian degeneration secondary to loss of associated grey matter [16]. Given that a number of disease processes likely conspire to cause structural brain changes in DM1, it is clear that a range of modalities should be employed in future neuroimaging studies. Future studies would also benefit from a longitudinal design. Of note, recent data from Gliem and colleagues [65] suggest the rate of structural brain change over time may be slow, and hence could be challenging to measure within the timescale of a typical clinical trial. Further, respiratory function was not specifically assessed as part of the present study. Sleep-disordered breathing is common in DM1 [66], and has been linked to various structural brain changes in other patient populations $[58,67,68]$, therefore detailed phenotyping of respiratory function including sleep studies should be considered as part of future neuroimaging work. Finally, the clinical impact of the structural changes identified were not explored as part of the present study. Correlation of structural measures with neuropsychological data would be an important extension of the work presented here, to identify how abnormal brain structure may relate to functional problems.

In summary, the present study highlights the potential for multi-site neuroimaging efforts to elucidate neuropathological features associated with inherited, rare neurological disorders, such as DM1. The pooled sample represents the largest case-control DM1 neuroimaging cohort at present. The study provided novel insights into the effects of DM1 on the human brain, by providing a detailed portrait of the relative volumetric changes occurring in specific regions. Several of the structural changes were directly cor- related with CTG repeat length, and occurred in structures with potential functional links to key features of the DM1 phenotype. These findings illuminate potential avenues for further research work to elucidate the molecular and neuroanatomical basis for central symptoms in DM1.

\section{CONFLICT OF INTEREST}

Ellen van der Plas- reports no disclosures Mark J. Hamilton- reports no disclosures

Jacob N. Miller- reports no disclosures

Timothy R. Koscik- reports no disclosures

Jeffrey D. Long- is a paid advisory board member for F. Hoffmann-La Roche Ltd, Wave Life Sciences USA Inc, Huntington Study Group, Mitoconix Bio Limited, a paid consultant for Vaccinex Inc and Azevan Pharmaceuticals Inc, and is funded by CHDI Inc, Michael J. Fox Foundation, and National Institutes of Health (USA).

Sarah Cumming- reports no disclosures

Julija Povilaikaite- reports no disclosures

Maria Elena Farrugia- reports no disclosures

John McLean- reports no disclosures

Ravi Jampana- reports no disclosures

Vincent A. Magnotta- reports no disclosures

Laurie Gutmann- reports no disclosures

Darren Monckton- has been a scientific consultant and/or received an honoraria or stock options from Biogen Idec, AMO Pharma, Charles River, Vertex Pharmaceuticals, Triplet Therapeutics, LoQus23, BridgeBio, and Small Molecule RNA. Darren Monckton is on the Scientific Advisory Board of the Myotonic Dystrophy Foundation, is a scientific advisor to the Myotonic Dystrophy Support Group and is a vice president of Muscular Dystrophy UK.

Peggy C. Nopoulos- reports no disclosures

\section{Study funding}

Work undertaken in Scotland was funded by Muscular Dystrophy UK (Ref: MC3/1073) and Chief Scientist Office (Ref: CAF/MD/15/01). MJH also received travel awards from the Clinical Genetics Society (UK) and the University of Glasgow (David Fleming-Brown Postgraduate Travel Scholarship) in relation to the work presented here. Research conducted at the Iowa site was funded by a grant from the National Institute of Neurological Disorders and Stroke (NINDS) (Ref: 5R01NS094387-02) and the Wyck Foundation. The funders did not have any role 
in the study design, data collection, analysis, or in preparing this report.

\section{ACKNOWLEDGMENTS}

The authors are grateful to the participants and their families for their cooperation with the study, and also to the team of research assistants who collected the data at the Iowa site. The authors thank the members of the Scottish Myotonic Dystrophy Consortium, in particular Dr Cheryl Longman and Dr. Bob Ballantyne, for facilitating work undertaken in Glasgow.

\section{SUPPLEMENTARY MATERIAL}

The supplementary material is available in the electronic version of this article: http://dx.doi. org/10.3233/JND-190397.

\section{REFERENCES}

[1] Brook JD, McCurrach ME, Harley HG, Buckler AJ, Church D, Aburatani H, Hunter K, Stanton VP, Thirion JP, Hudson T. Molecular basis of myotonic dystrophy: Expansion of a trinucleotide (CTG) repeat at the 3' end of a transcript encoding a protein kinase family member. Cell. 1992;69:385.

[2] Turner C, Hilton-Jones D. Myotonic dystrophy: Diagnosis, management and new therapies. Curr Opin Neurol. 2014;27:599-606

[3] Heatwole C, Bode R, Johnson N, et al. Patient-reported impact of symptoms in myotonic dystrophy type 1 (PRISM1). Neurology. 2012;79:348-57.

[4] Peric S, Stojanovic VR, Basta I, Peric M, Milicev M, Pavlovic S, Lavrnic D. Influence of multisystemic affection on health-related quality of life in patients with myotonic dystrophy type 1. Clin Neurol Neurosurg. 2013;115:270-5.

[5] Antonini G, Soscia F, Giubilei F, De Carolis A, Gragnani F, Morino S, Ruberto A, Tatarelli R. Health-related quality of life in myotonic dystrophy type 1 and its relationship with cognitive and emotional functioning. J Rehabil Med. 2006;38:181-5.

[6] Cup EHC, Kinébanian A, Satink T, Pieterse AJ, Hendricks HT, Oostendorp RAB, van der Wilt GJ, van Engelen BGM. Living with myotonic dystrophy; what can be learned from couples? A qualitative study. BMC Neurol. 2011;11:86.

[7] Gagnon C, Noreau L, Moxley RT, Laberge L, Jean S, Richer L, Perron M, Veillette S, Mathieu J. Towards an integrative approach to the management of myotonic dystrophy type 1 . J Neurol Neurosurg Psychiatry. 2007;78:800-6.

[8] Gagnon C, Meola G, Hébert LJ, Laberge L, Leone M, Heatwole C. Report of the second Outcome Measures in Myotonic Dystrophy type 1 (OMMYD-2) international workshop San Sebastian, Spain, October 16, 2013. Neuromuscul Disord. 2015;25:603-16.

[9] Bosco G, Diamanti S, Meola G, DM-CNS Group. Workshop Report: Consensus on biomarkers of cerebral involvement in myotonic dystrophy, 2-3 December 2014, Milan, Italy. Neuromuscul Disord. 2015;25:813-23.

[10] Okkersen K, Monckton DG, Le N, Tuladhar AM, Raaphorst $\mathrm{J}$, van Engelen BGM. Brain imaging in myotonic dystrophy type 1: A systematic review. Neurology. 2017;89:960-9.

[11] Minnerop M, Weber B, Schoene-Bake J-C, et al. The brain in myotonic dystrophy 1 and 2: Evidence for a predominant white matter disease. Brain. 2011;134:3530-46.

[12] Caso F, Agosta F, Peric S, Rakočević-Stojanović V, Copetti M, Kostic VS, Filippi M. Cognitive impairment in myotonic dystrophy type 1 is associated with white matter damage. PLoS One. 2014;9:e104697.

[13] Zanigni S, Evangelisti S, Giannoccaro MP, et al. Relationship of white and gray matter abnormalities to clinical and genetic features in myotonic dystrophy type 1 . Neuroimage Clin. 2016;11:678-85.

[14] Schneider-Gold C, Bellenberg B, Prehn C, Krogias C, Schneider R, Klein J, Gold R, Lukas C. Cortical and Subcortical Grey and White Matter Atrophy in Myotonic Dystrophies Type 1 and 2 Is Associated with Cognitive Impairment, Depression and Daytime Sleepiness. PLoS One. 2015; 10:e0130352.

[15] Baldanzi S, Cecchi P, Fabbri S, Pesaresi I, Simoncini C, Angelini C, Bonuccelli U, Cosottini M, Siciliano G. Relationship between neuropsychological impairment and grey and white matter changes in adult-onset myotonic dystrophy type 1. Neuroimage Clin. 2016;12:190-7.

[16] Ota M, Sato N, Ohya Y, Aoki Y, Mizukami K, Mori T, Asada T. Relationship between diffusion tensor imaging and brain morphology in patients with myotonic dystrophy. Neurosci Lett. 2006;407:234-9.

[17] Serra L, Petrucci A, Spanò B, et al. How genetics affects the brain to produce higher-level dysfunctions in myotonic dystrophy type 1. Funct Neurol. 2015;30:21-31.

[18] Cabada T, Iridoy M, Jericó I, Lecumberri P, Seijas R, Gargallo A, Gomez M. Brain Involvement in Myotonic Dystrophy Type 1: A Morphometric and Diffusion Tensor Imaging Study with Neuropsychological Correlation. Arch Clin Neuropsychol. 2017;32:401-12.

[19] Minnerop M, Gliem C, Kornblum C. Current Progress in CNS Imaging of Myotonic Dystrophy. Front Neurol. 2018;9:646.

[20] Wong LJ, Ashizawa T, Monckton DG, Caskey CT, Richards CS. Somatic heterogeneity of the CTG repeat in myotonic dystrophy is age and size dependent. Am J Hum Genet. 1995;56:114-22.

[21] Douniol M, Jacquette A, Cohen D, et al. Psychiatric and cognitive phenotype of childhood myotonic dystrophy type 1. Dev Med Child Neurol. 2012;54:905-11.

[22] Okkersen K, Buskes M, Groenewoud J, Kessels RPC, Knoop H, van Engelen B, Raaphorst J. The cognitive profile of myotonic dystrophy type 1: A systematic review and meta-analysis. Cortex. 2017;95:143-55.

[23] Morales F, Couto JM, Higham CF, et al. Somatic instability of the expanded CTG triplet repeat in myotonic dystrophy type 1 is a heritable quantitative trait and modifier of disease severity. Hum Mol Genet. 2012;21:3558-67.

[24] McKinstry B, Sullivan FM, Vasishta S, Armstrong R, Hanley J, Haughney J, Philip S, Smith BH, Wood A, Palmer CAN. Cohort profile: The Scottish Research register SHARE. A register of people interested in research participation linked to NHS data sets. BMJ Open. 2017;7: e013351.

[25] Mathieu J, Boivin H, Meunier D, Gaudreault M, Bégin P. Assessment of a disease-specific muscular impair- 
ment rating scale in myotonic dystrophy. Neurology. 2001;56:336-40.

[26] Tustison NJ, Avants BB, Cook PA, Zheng Y, Egan A, Yushkevich PA, Gee JC. N4ITK: Improved N3 bias correction. IEEE Trans Med Imaging. 2010;29:1310-20.

[27] Pierson R, Johnson H, Harris G, Keefe H, Paulsen JS, Andreasen NC, Magnotta VA. Fully automated analysis using BRAINS: AutoWorkup. Neuroimage. 2011;54:32836.

[28] Wang H, Suh JW, Das SR, Pluta JB, Craige C, Yushkevich PA. Multi-Atlas Segmentation with Joint Label Fusion. IEEE Trans Pattern Anal Mach Intell. 2013;35:611-23.

[29] Desikan RS, Ségonne F, Fischl B, et al. An automated labeling system for subdividing the human cerebral cortex on MRI scans into gyral based regions of interest. Neuroimage. 2006;31:968-80.

[30] Fortin J-P, Cullen N, Sheline YI, et al. Harmonization of cortical thickness measurements across scanners and sites. Neuroimage. 2018;167:104-20.

[31] Johnson WE, Li C, Rabinovic A. Adjusting batch effects in microarray expression data using empirical Bayes methods. Biostatistics. 2007;8:118-27.

[32] Koscik TR. 2018 ez.combat.

[33] Gomes-Pereira M, Bidichandani SI, Monckton DG. Analysis of unstable triplet repeats using small-pool polymerase chain reaction. Methods Mol Biol. 2004;277:61-76.

[34] Monckton DG, Wong LJ, Ashizawa T, Caskey CT. Somatic mosaicism, germline expansions, germline reversions and intergenerational reductions in myotonic dystrophy males: Small pool PCR analyses. Hum Mol Genet. 1995;4:1-8.

[35] Liu D, Johnson HJ, Long JD, Magnotta VA, Paulsen JS. The power-proportion method for intracranial volume correction in volumetric imaging analysis. Front Neurosci. 2014;8:356.

[36] Antonini G, Mainero C, Romano A, et al. Cerebral atrophy in myotonic dystrophy: A voxel based morphometric study. J Neurol Neurosurg Psychiatry. 2004;75:1611-3.

[37] Kassubek J, Juengling FD, Hoffmann S, et al. Quantification of brain atrophy in patients with myotonic dystrophy and proximal myotonic myopathy: A controlled 3-dimensional magnetic resonance imaging study. Neurosci Lett. 2003;348:73-6.

[38] Censori B, Provinciali L, Danni M, Chiaramoni L, Maricotti M, Foschi N, Del Pesce M, Salvolini U. Brain involvement in myotonic dystrophy: MRI features and their relationship to clinical and cognitive conditions. Acta Neurol Scand. 1994;90:211-7.

[39] Weber YG, Roebling R, Kassubek J, et al. Comparative analysis of brain structure, metabolism, and cognition in myotonic dystrophy 1 and 2. Neurology. 2010;74:1108-17.

[40] Pfefferbaum A, Mathalon DH, Sullivan EV, Rawles JM, Zipursky RB, Lim KO. A quantitative magnetic resonance imaging study of changes in brain morphology from infancy to late adulthood. Arch Neurol. 1994;51:874-87.

[41] Nopoulos PC. Huntington disease: A single-gene degenerative disorder of the striatum. Dialogues Clin Neurosci. 2016;18:91-8.

[42] Mehler MF, Gokhan S. Mechanisms underlying neural cell death in neurodegenerative diseases: Alterations of a developmentally-mediated cellular rheostat. Trends Neurosci. 2000;23:599-605.

[43] Haijma SV, Van Haren N, Cahn W, Koolschijn PCMP, Hulshoff Pol HE, Kahn RS. Brain volumes in schizophrenia: A meta-analysis in over 18000 subjects. Schizophr Bull. 2013;39:1129-38.
[44] Sellgren CM, Gracias J, Watmuff B, et al. Increased synapse elimination by microglia in schizophrenia patientderived models of synaptic pruning. Nat Neurosci. 2019;22: 374-85.

[45] Gallais B, Gagnon C, Mathieu J, Richer L. Cognitive decline over time in adults with myotonic dystrophy type 1: A 9-year longitudinal study. Neuromuscul Disord. 2017;27: $61-72$.

[46] Scahill RI, Frost C, Jenkins R, Whitwell JL, Rossor MN, Fox NC. A longitudinal study of brain volume changes in normal aging using serial registered magnetic resonance imaging. Arch Neurol. 2003;60:989-94.

[47] Aylward EH, Nopoulos PC, Ross CA, et al. Longitudinal change in regional brain volumes in prodromal Huntington disease. J Neurol Neurosurg Psychiatry. 2011;82:405-10.

[48] Saper CB, Scammell TE, Lu J. Hypothalamic regulation of sleep and circadian rhythms. Nature. 2005;437:1257-63.

[49] Itoh K, Mitani M, Kawamoto K, Futamura N, Funakawa I, Jinnai K, Fushiki S. Neuropathology does not Correlate with Regional Differences in the Extent of Expansion of CTG Repeats in the Brain with Myotonic Dystrophy Type 1. Acta Histochem Cytochem. 2010;43:149-56.

[50] Jinnai K, Mitani M, Futamura N, Kawamoto K, Funakawa I, Itoh K. Somatic instability of CTG repeats in the cerebellum of myotonic dystrophy type 1. Muscle Nerve. 2013;48: 105-8.

[51] Jiang H, Mankodi A, Swanson MS, Moxley RT, Thornton CA. Myotonic dystrophy type 1 is associated with nuclear foci of mutant RNA, sequestration of muscleblind proteins and deregulated alternative splicing in neurons. Hum Mol Genet. 2004;13:3079-88.

[52] Caillet-Boudin M-L, Fernandez-Gomez F-J, Tran H, Dhaenens C-M, Buee L, Sergeant N. Brain pathology in myotonic dystrophy: When tauopathy meets spliceopathy and RNAopathy. Front Mol Neurosci. 2014;6:57.

[53] Yoshimura N, Otake M, Igarashi K, Matsunaga M, Takebe $\mathrm{K}$, Kudo H. Topography of Alzheimer's neurofibrillary change distribution in myotonic dystrophy. Clin Neuropathol. 1990;9:234-9.

[54] Ladd AN. CUG-BP, Elav-like family (CELF)-mediated alternative splicing regulation in the brain during health and disease. Mol Cell Neurosci. 2013;56:456-64.

[55] Hernández-Hernández O, Guiraud-Dogan C, Sicot G, et al. Myotonic dystrophy CTG expansion affects synaptic vesicle proteins, neurotransmission and mouse behaviour. Brain. 2013;136:957-70.

[56] Anvret M, Ahlberg G, Grandell U, Hedberg B, Johnson K, Edström L. Larger expansions of the CTG repeat in muscle compared to lymphocytes from patients with myotonic dystrophy. Hum Mol Genet. 1993;2:1397-400.

[57] Dhaenens CM, Tran H, Frandemiche M-L, et al. Missplicing of Tau exon 10 in myotonic dystrophy type 1 is reproduced by overexpression of CELF2 but not by MBNL1 silencing. Biochim Biophys Acta. 2011;1812:732-42.

[58] Macey PM, Prasad JP, Ogren JA, Moiyadi AS, Aysola RS, Kumar R, Yan-Go FL, Woo MA, Albert Thomas M, Harper RM. Sex-specific hippocampus volume changes in obstructive sleep apnea. Neuroimage Clin. 2018;20:305-17.

[59] Nobis WP, Schuele S, Templer JW, Zhou G, Lane G, Rosenow JM, Zelano C. Amygdala-stimulation-induced apnea is attention and nasal-breathing dependent. Ann Neurol. 2018;83:460-71.

[60] Pincherle A, Patruno V, Raimondi P, et al. Sleep breathing disorders in 40 Italian patients with Myotonic dystrophy type 1. Neuromuscul Disord. 2012;22:219-24. 
[61] Cunningham WA, Brosch T. Motivational Salience: Amygdala Tuning From Traits, Needs, Values, and Goals. Curr Dir Psychol Sci. 2012;21:54-9.

[62] van der Velden BG, Okkersen K, Kessels RP, Groenewoud J, van Engelen B, Knoop H, Raaphorst J. Affective symptoms and apathy in myotonic dystrophy type 1 a systematic review and meta-analysis. J Affect Disord. 2019;250:260-9.

[63] Babaev O, Piletti Chatain C, Krueger-Burg D. Inhibition in the amygdala anxiety circuitry. Exp Mol Med. 2018;50:18.

[64] Fukuda H, Horiguchi J, Ono C, Ohshita T, Takaba J, Ito K. Diffusion tensor imaging of cerebral white matter in patients with myotonic dystrophy. Acta Radiol. 2005;46:104-9.

[65] Gliem C, Minnerop M, Roeske S, et al. Tracking the brain in myotonic dystrophies: A 5-year longitudinal follow-up study. PLoS One. 2019;14:e0213381.
[66] Laberge L, Bégin P, Dauvilliers Y, Beaudry M, Laforte M, Jean S, Mathieu J. A polysomnographic study of daytime sleepiness in myotonic dystrophy type 1 . J Neurol Neurosurg Psychiatry. 2009;80:642-6.

[67] Morrell MJ, Jackson ML, Twigg GL, et al. Changes in brain morphology in patients with obstructive sleep apnoea. Thorax. 2010;65:908-14.

[68] Zuurbier LA, Vernooij MW, Luik AI, Kocevska D, Hofman A, Whitmore H, Ikram MA, Tiemeier H. Apneahypopnea index, nocturnal arousals, oxygen desaturation and structural brain changes: A population-based study. Neurobiology of Sleep and Circadian Rhythms. 2016;1:1-7. 